\title{
Das Göttweiger Interstadial, ein zweiter Leithorizont der letzten Vereisung
}

\author{
Von Hugo Gross, Bamberg
}

\section{Mit 1 Tabelle}

Zusammenfassung: Die Definition des Begriffs Interstadia 1 muß auf Grund neuer pollenanalytischer Untersuchungen von Bildungen des Alleröd-Interstadials dahin abgeändert werden, daß die Amplitude des interstadialen Klimas von subarktisch bis temperiert reichte, daß also auch Bewaldung und Bodenbildung in einem Interstadial möglich waren; ein nennenswertes Eichenmischwald- und Hasel-Maximum fehlten; auf die Zeitdauer kommt es nicht an. Die paläofaunistische und -floristische, die pedologische, sedimentanalytische und archäologische Untersuchung jungpleistozäner Lößprofile im südöstlichen semi-ariden Mitteleuropa hat ergeben, daß die ca. $0,5-1 \mathrm{~m}$ mächtige $\mathrm{Gö} \mathrm{t} \mathrm{tw}$ e i ger L e i m e n z o ne in einem schätzungsweise 15000 Jahre langen temperierten Interstadial mit Waldsteppenklima entstanden ist, das zwei weitgehend selbständige Würmstadiale (Altw ürm mit Moustérien und $\mathrm{Hauptw}$ i r m $\mathrm{mit}$ Jungpaläolithikum) voneinander trennt; zum Göttweiger Interstadial gehören auch die Schwankungen von $S$ p i e $z$ und $L$ a u f e $n$. In Höhlen hat dieses Interstadial eine meist ca. $20 \mathrm{~cm}$ mächtige Höhlenlehmschicht zwischen den Schichten mit Moustérien und Jungpaläolithikum durch intensive chemische Verwitterung von Kalkschutt erzeugt. In Nordamerika trennt das gleichzeitige, ca. 16000 Jahre lange Sidne y-Inte rs t a dial A $1 \mathrm{t}-$ und $\mathrm{H}$ a u p $\mathrm{t}$ is consin.

$\mathrm{Summary}$ : On the ground of recent pollen-analytical studies concerning the Alleröd Interstadial, the definition of the notion "in te rstadial " is to be corrected by the amendment that it implies climates from subarctic to temperate, thus the possibility, too, of forest growth and soil formation; a maximum worth mentioning of hazel and mixed oak forest were absent; the duration is irrelevant. The study of Upper Pleistocene loess sections in the semi-arid countries of southeastern Central Europe applying palaeofaunistic, palaeofloristic pedological, sediment-analytical, and archaeological researches suggested that the Göttweig fossil soil $(0,5$ to $1 \mathrm{~m}$ in thickness) of those loess sections must have been formed during a temperate interstadial with forest-steppe climate for around 15000 years (estimated), which has separated two fairly independent Würmian stadials (A n c i e $\mathrm{t}$ W ürm wi th Mousterian and $M$ a in Würm with Upper Palaeolithic); the Göt tweig interstadial includes the Spiez and $L$ a u e n interstadials. Within cave deposits, it has produced a cave loam stratum of mostly $20 \mathrm{~cm}$ thickness by intensive chemical disintegration of limestone debris. In North America, the $\mathrm{S}$ idney interstadial of ca. $16000 \mathrm{yr}$ in length is the correlative of the Göttweig interstadial, it separates an $\mathrm{E}$ a rly W is con s in stadial from the $\mathrm{M}$ a in W is con$s$ i n glaciation.

Die trotz jahrzehntelanger Arbeit noch immer nicht allgemein befriedigend gelungene Lösung des Problems der Gliederung und Chronologie des Jungpleistozäns in Europa ist nur von einer a $1 \mathrm{le}$ in Frage kommenden Wissenschaften heranziehenden Auswertung der $\mathrm{L}$ ö $ß \mathrm{p}$ r o f i l e in den semi-ariden Klimagebieten des südöstlichen Mitteleuropa (östliches Osterreich und Tschechoslowakei) zu erwarten, wo die Lößstratigraphie am deutlichsten ausgeprägt ist und Abspülung sowie Solifluktion eine sehr viel geringere Rolle als im W spielten; denn im Löß sind die großen säkularen Klimaänderungen des Pleistozäns ohne Verzögerung (wie bei den Moränen) im Wechsel von fossilen Böden, Fließerden und nicht verlehmtem Löß registriert. Weniger deutlich ist naturgemäß dieser Klimawandel in den Höhlenschichtenfölgen zu erkennen; aber wenn sie mit der Sedimentanalyse von R. LAIS (1941), ferner faunistisch und archäologisch so sorgfältig untersucht worden sind wie z. B. die Weinberghöhlen von Mauern (L. F. Zotz 1955), können sie mit den jungpleistozänen Lößprofilen des genannten Gebiets parallelisiert werden; das ist sehr wichtig, weil sie für die Erforschung der jungpleistozänen Biotope viel mehr Befunde liefern als die meisten Lößprofile. Die Fluß- und Stromterrassen im Periglazial kommen in diesem Zusammenhang so lange nicht in Frage, wie allen Ernstes von geologischer Seite auf der N i e d e r terrasse Lößpakete von 9 bzw. 11 m Mächtigkeit mit 2 (zwei) fossilen Böden gefunden werden! 
Das Problem der Gliederung und Chronologie des Jungpleistozäns stand im Vordergrund des Interesses auf dem IV. INQUA-Kongreß in Rom und Pisa 1953 und auf den Tagungen der Deutschen Quartärvereinigung 1953, 1954 und vor allem 1955; besonders wichtig waren die von den österreichischen Geologen glänzend vorbereiteten und geleiteten Exkursionen der Tagung von 1955 von der Salzach bis zur March, in erster Linie zum Studium von Terrassen und Lößprofilen. Die im Anschluß daran in der Geologischen Bundesanstalt in Wien veranstaltete ausgedehnte Diskussion führte leider zu keiner Einigung, da die Ansichten über die Natur und Zeitstellung der G ö t t w e ig e r B o d e n b ild u n g im Lößprofil geteilt blieben, von deren zeitlicher Einstufung, wie F. BRANDTNER (1950) richtig erkannt hat, jede Diluvialchronologie abhängt.

Schuld an dieser Uneinigkeit ist die Verwendung eines längst überholten Interstadialbegriffs und die unmögliche Pleistozängliederung von J. BAYER (1927), der als erster die stratigraphische Bedeutung der Leimenzone im Lößprofil erkannt hat, die er G ö t weiger Verlehmungszone nannte; als Biglazialist deutete er in seiner jungdiluvialen Eiszeit (= Riß + Würm) das Riß-Würm-Interglazial von A. PencK als Schwankung (Interstadial) um und stufte in diese die Göttweiger Verlehmungszone ein, deren Bildungszeit er als A u rig n a c-S ch w a n k u ng bezeichnete, da in ihrer zweiten Hälfte in Mitteleuropa das Aurignacien erschien.

In der Regel ist aber die Göttweiger Bodenbildung wegen ihrer Mächtigkeit für eine in te rg l a z i a le Bildung gehalten worden. G. Götzinger $(1936,1938)$ stellte sie ins RW-Interglazial, ebenso J. Büdel (1950), F. WeIDENBACH $(1952,1955)$ sowie in Wien 1955 J. Fink und H. Freising (der sie 1949 und 1951 ausdrücklich noch als „W I-W II-Warmzeit", später als „letzte Warmzeit" bezeichnet hatte). Das RW-Interglazial im Sinne von A. PeNCK stellten J. Büdel (1953) und F. WeIDENBACH (1955) zwischen Alt-bzw. Mittelund Jungriß, obwohl das RW-Interglazial im Sinne von A. PENCK das letzte Vollinterglazial mit der letzten Antiquus-Fauna ist. Nach H. Gams $(1935,1954)$ entspricht die Göttweiger Leimenzone dem F-Interglazial zwischen Präwürm und Würm, d. h. dem temperierten Abschnitt des RW-Interglazials im Sinne von K. Jessen \& V. Milthers (1928). Als erste Würmschwankung, also Interstadial, wurde die Bildungszeit der Göttweiger Leimenzone zuerst von R. GrahmanN $(1937,1952,1956)$ und R. LAIs (1951) aufgefaßt, ebenso von F. E. ZeUner $(1952,1954)$ als „temperate interstadial L. Gl. 1/2, quite as temperate as the Postglacial" zwischen Warthe-Stadium und Würm (im Sinne von A. PENCK), als würminterstadiale Wärmezeit F (= W I/W II) von F. BRANDTNER (seit 1950) und als „starkes Interstadial W 1/2“ von Fr. Prošex \& V. LožEK (1955).

Es sind nun zunächst zwei Fragen zu beantworten: 1. Welche Zeitstellung hat die Göttweiger Bodenbildung? (F. BRANDTNER 1954 zieht wegen der Vollständigkeit ihres fossilen Bodens die Bezeichnung Fellabrunner Bodenkomplex, J. Fink 1954 den Namen Stillfrieder Komplex vor; es kommt aber in erster Linie auf die bei Göttweig erhalten gebliebene Verlehmungszone an.) 2. Ist diese Bodenbildung in ein Interstadial oder in ein Interglazial einzustufen?

Die einzige Möglichkeit einer z u ve r läss ig e n Datierung bietet die pedologische, paläontologische u n d archäologische Auswertung der jungpleistozänen Lößstratigraphie des oben genannten Gebietes, wie sie F. BRANDTNER (1954) seit Jahren mit seinen Mitarbeitern auf Grund eines sehr umfangreichen Beobachtungsmaterials in Nieder-OOsterreich und Fr. ProšEK \& V. LožEK (1955) und andere tschechische Forscher im östlichen Nachbargebiet ausgeführt haben. Dort sind in der Regel unter dem rezenten Boden drei begrabene fossile Böden festgestellt worden, die besonders gut an alten Lößwänden zu unterscheiden sind: der I. (von oben) ist die schwache graubraune $\mathrm{P}$ a u dorf e r B od e n bil d ung (im Mittel 0,61 m mächtig nach R. LAIS 1951), der II. die viel bedeutendere G ö t $\mathrm{t}$ w e i g e r B o d e n bil d u n g mit (nach F. BRANDTNer durchschnittlich 0,50 m, maximal $1 \mathrm{~m}$ mächtiger) hellrötlich-brauner Verlehmungszone mit intakter Lößstruktur und, mei- 
stens durch dünne gelbe Lößlagen getrennt, 1-3 Schwarzerdeschichten im Hangenden, die keinen Oberboden A der Verlehmungszone darstellen (R. LaIs 1951, F. BRANDTNER 1954, J. Fink 1954, 1955) und der III. die K r e m s e r B o d e n b il d u n g mit A-Horizont und fast ziegelroter, bis zu $2 \mathrm{~m}$ mächtiger Verlehmungszone mit völlig vernichtetem ursprünglichem Lößgefüge. Die noch mächtigere $(\mathrm{ca} .3 \mathrm{~m}$ ) rotbraune Verlehmungszone des Laaerbergs in Wien (Rudolfsziegelöfen) ist nach dem paläontologischen Befund eine Bodenbildung des MR-Interglazials (H. KüPPER 1955). Schon hiernach ist anzunehmen, daß die Kremser Bodenbildung, wie es schon H. Freising $(1949,1951)$ und F. Brandtner (1950, 1954) getan haben, ins RW-Interglazial zu stellen ist; dafür, daß der unmittelbar darunter liegende Löß wirklich Riß-Löß ist, sprechen die kopfgroßen Lößkindl, worauf wiederholt R. Grahmann aufmerksam gemacht hat. Dann muß aber die G ö t t w e i g e r B odenbildung w ürmeiszeit lich sein.

Den Beweis dafür erbrachten tschechoslowakische Untersuchungen der Lößstratigraphie mit Berücksichtigung der Schneckenfauna und paläolithischen Funde. Besonders überzeugend ist das Lößprofil von Zamarovce bei Trentschin in der Slowakei (ca. $220 \mathrm{~m} \mathrm{M} \mathrm{H}$, Jahresmittel der Temperatur $8,8^{\circ} \mathrm{C}$, des Niederschlags $677 \mathrm{~mm}$ ) nach Fr. ProšEk \& V. LožEK (1955). Der III. fossile Boden mit rotbrauner Verlehmungszone vom Typ des braunen Waldbodens ist auf Grund der sehr „warmen“ Banatica-Fauna (unmittelbar unter diesem Boden) mit den Leitformen Helicigona banatica Rossm. und Cepaea rindobonensis ins RW-Interglazial zu stellen, denn diese thermophile Schneckenfauna ist nach den genannten Autoren ein Äquivalent der Antiquus-Fauna, da sie aus dem RW-Interglazial in Thüringen (Burgtonna, Ehringsdorf), dem Harzvorland (Osterode) und von 3 Stellen $\mathrm{N}$ von Prag bekannt und nicht jünger als dieses Interglazial ist. Der III. fossile Boden im Lößprofil von Zamarovce kann also nur die Kremser Bodenbildung und daher der II. fossile Boden, die G ö t t w e i g e r B o d e n bil d u n g, w ü r me is z e it li ch sein. Die spärliche Schneckenfauna dieses II. fossilen Bodens ist nicht so thermophil wie die des III., die Färbung der Verlehmungszone braungrau bis braun; die mittlere Schicht (4b) enthält Szeletien-Artefakte in primärer Lagerung. Das Szeletien ist eine Fazies des nordungarisch-slowakischen Protosolutréen (das zum Praesolutréen II von L. F. ZoTz 1955 gehört), typologisch mit dem dortigen Spätmoustérien verwandt, das im Liegenden der Schichten mit Protosolutréen in Tata, in der Kiskevélyer-, Subalyuk- und Szelim-Höhle festgestellt worden ist (G. Freund 1952); die Anfänge des ungarischen Protosolutréen wie des mitteleuropäischen Praesolutréen lagen im Moustérien. Das voll entwickelte Moustérien ist aber jünger als die Riß-Eiszeit, seine Blütezeit fällt in den ersten Vorstoß der Würm-Eiszeit (W I der Prähistoriker), wie P. WoldSTEDT in seinem Aufsatz in diesem Jahrbuch ausführlich begründet hat. Die Hauptentwicklung des ungarischen Protosolutréen und des mitteleuropäischen Praesolutréen dürfte sich aber im 1. Würm-Interstadial, d. h. in der Zeit der Göttweiger Bodenbildung, abgespielt haben; während dieser Zeit muß allmählich ein Kontakt mit dem von $\mathrm{O}$ vordringenden Aurignacien eingetreten sein (G. FreUND 1952, L. F. ZotZ 1955).

In den leider nur ausnahmsweise nicht (z. B. durch Füchse, Dachse und Raubgräber) gestörten Schichtenfolgen in Höhlen kann mit Hilfe der von R. LAIs (1941) eingeführten Sedimentanalyse, wenn sie mit den nötigen Kautelen (Berücksichtigung der Faunen- und Kulturabfolge) angewandt wird, trotz den von K. BRUNNACKeR (1956) vorgebrachten Bedenken eine Parallelisierung mit spätpleistozänen Lößprofilen vorgenommen werden. Daß das möglich ist, beweist die stattliche Monographie der Weinberghöhlen von Mauern (zwischen Ingolstadt und Donauwörth) von L. F. ZoTz (1955) auf Grund der umfangreichen faunistischen Untersuchung durch F. HeLler und der ebenso sorgfältigen Höhlensediment-Analyse von G. Freund. F. Heller fand im Gegensatz zu A. Bohmers $(1939,1944)$ keine Spur einer interglazialen Fauna und nimmt wie L. F. Zotz und G. Freund an, daß nur die basale sterile ockergelbe Lehmschicht J aller Wahrscheinlichkeit 
nach ins RW-Interglazial zu stellen ist. G. Freund und L. F. Zotz fanden unter der sehr blattspitzenreichen Schicht $F_{1}$ eine $15-20 \mathrm{~cm}$ mächtige sterile ockergelbe Lehmschicht $\mathrm{F}_{2}$ (sehr ähnlich der interglazialen Schicht J, also ein Produkt intensiver chemischer Verwitterung von Kalkschutt) mit ganz vereinzeltem feinem kantengerundetem Kalkschutt über den Höhlenlehmschichten $\mathrm{G}$ und $\mathrm{H}$ mit viel kantengerundetem Grobschutt und Steinen (Frostbruchstraten vom 1. Würmstadial); sie stellen die Höhlenlehmschicht $F_{2}$ mit Recht ins 1. Würminterstadial, d.h. in die Zeit der Göttweiger Bodenbildung; die von A. Bohmers mit D bezeichnete Schicht, von H. Freising (1949) mit der Göttweiger Bodenbildung parallelisiert, ist vielleicht eine sehr undeutliche Entsprechung des 2. Würminterstadials, d. h. der Paudorfer Bodenbildung im Lößprofil (L. F. Zotz 1955). Ferner eignet sich für eine solche Parallelisierung die von G. RIEK (1934) stratigraphisch und archäologisch und von U. Lehmann (1954) faunistisch untersuchte Schichtenfolge in der Vogelherdhöhle, deren letztinterglazialer Beginn (RW) ein Molar von Elephas antiquus und JungAcheuléen-Artefakte an der Basis beweisen. R. WETZEL (1954) hatte schon lange bei seinen äußerst sorgfältigen Untersuchungen der Schichtenfolgen anderer Höhlen im Lonetal die Schlüsselstellung einer dünnen gelben Lehmschicht zwischen den Frostbruchstraten mit Moustérien im Liegenden und Aurignacien im Hangenden für die geochronologische Auswertung von Höhlenprofilen erkannt; nach der neu erschienenen Monographie von L. F. ZотZ (1955) kann kein Zweifel daran bestehen, daß diese Höhlenlehmschicht im 1. Würminterstadial, d.h. in der Zeit der Göttweiger Bodenbildung, entstanden ist, daß also diese Bodenbildung nicht in das RW-Interglazial, sondern in eine bedeutende Wärmeschwankung der Würmeiszeit einzustufen ist.

Die Mächtigkeit dieser Bodenbildung hat bekanntlich zahlreiche Geologen dazu verleitet, sie für zweifellos interglazial zu halten. Die Ursache dafür ist die längst überholte von A. PENCK gegebene Definition des Begriffs Interstadial als „Zeit einer großen Gletscherschwankung, jedoch ohne Wiederbewaldung und Bodenbildung". Diese Definition gilt aber nur für einen vorübergehenden Eisrückgang (Oszillation), der nicht klimatisch bedingt zu sein braucht. Denn für das bekannteste Interstadial, das etwas über 1000jährige Alleröd-Interstadial, ist Bewaldung und (geringmächtige) Bodenbildung seit seiner Entdeckung bekannt. In dieser Wärmeschwankung im Spätglazial war der Rand des nordischen Inlandeises nach Südschweden und Südfinnland abgerückt und strebte der späteren Randlage des Salpausselkä-Stadiums zu; das alpine Inlandeis war kurz vor diesem Interstadial in Tirol bis über $1800 \mathrm{~m}$, in den französischen Westalpen bis über $2100 \mathrm{~m} \mathrm{M} \mathrm{H}$ abgeschmolzen (H. Gross 1954). Bis vor kurzem hat man auf Grund der Baumpollendiagramme das Klima des Alleröd-Interstadials in Mitteleuropa für gemäßigt "subarktisch“ gehalten; neuerdings hat aber Johs. Iversen (1954) auf Grund neuer Pollenfunde festgestellt, daß in seiner zweiten Hälfte in Dänemark das Klima t e m p e r i e r t war (Temperatur-Mittel im Juli $+13-14^{0} \mathrm{C}$, im Januar nicht tiefer als $-8^{0} \mathrm{C}$ ). Wesentlich ist, daß bei einer interstadialen Ablagerung ein EMW- und ein Corylus-Maximum wie in einem interglazialen oder im postglazialen Klimaoptimum fehlen, daß kälteempfindliche Holzarten höchstens vereinzelt in geschützten Lagen im S auftraten (Abies, Fagus und Carpinus), falls ihr Pollen nachweislich nicht sekundär ist, daß pleistozäne thermophile Tierund Pflanzenarten (Antiquus- und Banatica-Fauna, Brasenia und Dulichium)nicht auftraten und daß die Inlandeismassen (besonders im N) noch nicht bis auf den Umfang der heutigen Vergletscherung zurückgeschmolzen waren; das Klima kann subarktisch bis temperiert gewesen sein; die Dauer ist belanglos für die Begriffsbestimmung.

Die sehr spärliche Schneckenfauna (mit Orcula dolium Drap., Chondrula tridens Müll. und Arianta arbustorum L.) des II. fossilen Bodens im Lößprofil von Zamarovce ist nach Fr. Prošé \& V. LožEx (1955) „warm“, aber weniger thermophil als die BanaticaFauna des III. fossilen Bodens (Kremser Bodenbildung des R W-Interglazials). W. KüHNELT (1938) gibt für den Wiener Raum auch Eulota fruticum und Cepaea vindobonensis für die Göttweiger Bodenbildung an. 
In den Höhlenprofilen kommt nirgends nördlich der Alpen in der Schicht zwischen den Frostbruchstraten mit Moustérien bzw. Aurignacien eine typische interglaziale Fauna (mit Elephas antiquus und Rhinoceros mercki) vor, wohl aber überwiegt in ihr eine klimatisch indifferente zusammen mit einer temperierten Wald-und (besonders im O) $\mathrm{S} t$ e p p e $\mathrm{n} f \mathrm{a}$ n a den Anteil hochglazialer Faunenelemente (Mammut, Wollnashorn, Ren, Moschusochs, Lemminge). Klimatisch indifferente Arten sind: Höhlenlöwe, Höhlenhyäne und vor allem der Höhlenbär (in der Protosolutréen-Schicht der Szeleta-Höhle 99\% der Gesamtfauna!), der wie der Riesenhirsch geradezu als Leitform dieser Wärmeschwankung bezeichnet werden kann. Das Waldfaunenelement der gemäßigten Zone war damals mit folgenden Arten vertreten: Wildschwein, Steinbock, Gemse, Ur, Waldwisent (Bison bonasus), Edelhirsch, Elch, Wildkatze, Fuchs, Wolf, Dachs, Baummarder, Braunbär, Biber, Schneehase, das Steppenelement mit: Steppenwisent (B. priscus), Saiga-Antilope, Wildpferden, Steppenmurmeltier (Bobak), Hamster, Pfeifhase, Steppenspringer, Ziesel u. a., besonders artenreich in der SubalyukHöhle im Bükk-Gebirge (Ungarn) in der Neandertaler-Fundschicht (M. MоттL 1938), die unverständlicherweise trotz den stratigraphischen und faunistischen Befunden immer noch ins RW-Interglazial gestellt wird. Die genannten Steppentiere sind Arten des g e mä $\beta$ i g t e n Klimas, der Steppenspringer geht in O-Russland nicht über den $52^{\circ} \mathrm{N}$ hinaus, worauf schon A. NeHring (1890) aufmerksam gemacht hat, nur der Pfeifhase steigt ins Randgebiet der Tundra hinab; diese erdbewohnenden Steppennager können natürlich nicht in Dauerfrostboden leben, was von manchen Lößspezialisten nicht bedacht wird.

Die Fauna der 1. Würmschwankung läßt also auf ein temperiertes Waldsteppenklima schließen mit einer Pflanzendecke, die wenigstens in Trockengebieten und sonst zeitweise eine Gras- und Buschsteppe war, die vornehmlich an feuchten Stellen, besonders in Flußtälern, von Wald durchsetzt war. In dieser Zeit begann die eurosibirische Steppenfauna mit dem Altmenschen (Homo sapiens diluvialis), worauf schon H. GaMs (1935) hingewiesen hat, nach Westen zu fluten, um im Spät-Aurignacien die Pyrenäen zu erreichen (sogar die Saiga-Antilope!).

Hauptsächlich in diese Zeit fällt sehr wahrscheinlich auch das Vordringen eurosibirischer Steppenpflanzen nach Westen, von denen manche die jungpaläolithische Hauptwürmeiszeit in Mitteleuropa überdauert haben dürften; es war höchst wahrscheinlich die Zeit der großen diluvialen Steppen Mitteleuropas im Sinne von A. Nehring (1890) und M. VAHL (1902). Makroskopische Baumreste liegen in vielen Höhlen besonders des südlichen Mitteleuropa in Form von Holzkohle vor; ihre vegetationsgeschichtliche Auswertung muß aber in Betracht ziehen, daß sie teilweise aus hangenden postglazialen Schichten durch wühlende Tiere in die würmeiszeitlichen verschleppt sein können. In Mitteleuropa sind nachgewiesen: Zirbelkiefer (Pinus cembra), Bergkiefer ( $P$. mugo), Waldkiefer ( $P$. silvestris), Fichte (Picea Abies, im $\mathrm{O}$ auch $P$. obovata), Lärche (im $\mathrm{O}$ Larix polonica). Die Laubholzkohlefunde sind auch im $S$ verschwindend gering: Birke, Eberesche, Eiche, Hasel, Ulme (wohl U. montana), die aber heute in O-Europa bis zum $60^{\circ} \mathrm{N}$, teilweise bis zum $63^{\circ} \mathrm{N}$ nordwärts gehen ('TH. Schmucker 1942); die Eberesche kommt sogar im südlichsten Grönland vor und ist auch für die spätglaziale Böllingschwankung pollenanalytisch nachgewiesen (JoHs. IVERSEN 1954).

Auf Grund pollenanalytischer Untersuchung humoser Schichten der Göttweiger Bodenbildung in Nieder-O'Osterreich hat F. BRANDTNER (1950) folgende Arten angegeben: Kiefer, Fichte, Birke, Weide, Erle, Hasel, Ulme, Eiche und Linde; eine solche Untersuchung dürfte aber nur in besonders günstigen Fällen möglich sein. R. SснütRumpF (1944), der die Aussichtslosigkeit einer pollenanalytischen Untersuchung von Höhlenlehmen festgestellt hat, untersuchte im Wellheimer Tal vor den Weinberghöhlen von Mauern pollenanalytisch eine unter 2,95 m mächtiger Lößdecke liegende fast $5 \mathrm{~m}$ mächtige graue Tonschicht 
mit humosen Zwischenschichen und an der Basis mit 0,90 m mächtiger Waldtorfschicht auf rißeiszeitlichen Donauschottern. Die Parallelisierung der Schichtenfolge von $400 \mathrm{~cm}$ bis $800 \mathrm{~cm}$ auf Grund der Schwermineralanalyse mit den von A. BoHmers (1944) fälschlich ins RW-Interglazial gestellten Höhlenschichten F bis H ist, wie G. FREUND (1952) bereits ausgeführt hat, unmöglich. Das von Pinus, Picea, Betula und Salix beherrschte Pollendiagramm mit kleinen Mengen von Pollen wärmeliebender Holzarten ohne Fagus (Eiche, Ulme, Erle, Hasel, Weißbuche zusammen in der untersten humosen Schicht maximal 5\%), durchgehender Weidenpollenkurve und fast zusammenhängender Hippophä̈-Kurve gilt sehr viel eher für die 1. Würmschwankung, die im Lößprofil die Göttweiger Bodenbildung hervorgebracht hat. Ob das von F. BRANDTNER (1949) pollenanalytisch untersuchte Moor von Roggendorf bei Melk (Nieder-Osterreich) aus dieser Zeit stammt, obwohl eine geschlossene primäre Lößdecke fehlt, kann erst durch die geplante $\mathrm{C}^{14}$-Datierung einer Torfprobe entschieden werden; die für dieses Moor angegebene Zirbelkiefer auf Torf ist fraglich, da dieser Baum bisher auf Torf nicht bekannt ist. Nördlich von Ravensburg (Württemberg) hat A. PENCK eine intramoränale Moorbildung vom Karrestobel als würminterstadial bezeichnet; eine Probe von hier hat Herr Oberlandesgeologe Dr. WEIDENвАС dankenswerterweise für die $\mathrm{C}^{14}$-Datierung besorgt. Als pollenfrei erwies sich sowohl der Seeton unter dem Laufener Schotter (der von A. PENCK bekanntlich zuerst in seine würmeiszeitliche Laufenschwankung, später aber ins RW-Interglazial gestellt worden ist) als auch ungebändenter interglazial aussehender Mergel einer Bohrung (Furth 1) bei Tittmoning unter Würmmoräne aus 106,20 m bis $106,40 \mathrm{~m}$ Teufe, die ich von Herrn Oberregierungsrat Dr. Traub erhalten habe. Die von P. Beck $(1938,1954,1955)$ in der Schweiz bei Thun im Glütschtal und Kanderdurchstich festgestellte $\mathrm{S}$ p i e z e r S c h w a n k u n g mit 10-25 m mächtigen Schottern in $600 \mathrm{~m} \mathrm{MH}$ mit einem Mammut-Molar von aurignacienzeitlichem Typ, mit Eichenholz und 3-10 (-20) cm mächtiger Schieferkohlenschicht (im Glütschtal; vgl. C. Rathjens 1951, Fig. 6) zwischen zwei Würm-Moränen (W I und W II nach P. ВECK) ist höchst wahrscheinlich ebenfalls in die 1. Würmschwankung einzustufen; die $\mathrm{C}^{14}$-Datierung erfolgt in Bern. Die pollenanalytische Untersuchung dieser Schieferkohle und der im Profil angrenzenden pollenführenden Schichten von 2 Fundstellen durch W. LüDr (1953, S. 39-43), der sie eher ins RW-Interglazial stellen möchte, ergab ein im allgemeinen übereinstimmendes Waldbild: vorherrschend Kiefer und Fichte mit wechselnder Dominanz, niedrige Abies-, Alnus- und Betula-Pollenwerte, vereinzelt Hasel, Eiche, Carpinus und Fagus (sekundäre Pollenzufuhr?). W. LüDr (1953) möchte noch folgende Schweizer Vorkommen pollenführender Schichten in dieses Interstadial stellen: GenfCartigny, Chancy, Signal de Bougy, Mutten, Niederweningen (mit Mammutresten!) und Weiherbach; in diesen Bildungen treten außerdem in kleinen Mengen Pollen von Tilia, Ulmus, Pinus cembra und Larix auf.

Besonders wichtig ist in archäologischer wie in geologischer Hinsicht die berühmte Potočka-Höhle, die höchstgelegene jungpaläolithische Station in den Alpen (J. BAYER 1929, S. Brodar 1938), die in 1700 m M H am SW-Hang des 1930 m hohen Gebirgsstockes Olschewa in den Ostkarawanken in Jugoslawien unweit der S-Grenze des alpinen Inlandeises der letzten Eiszeit liegt. Die Steingeräte dieser Bärenhöhle (bis 1938 ca. 1500 Höhlenbären festgestellt!) tragen allgemein Aurignacien-Charakter (mittleres oder entwickeltes Aurignacien nach S. Brodar 1938, G. Freund 1952 und K. J. NarR 1954), außerdem kommen auch Geräte von moustérienartigem Habitus vor, die an das „alpine Paläolithikum" von E. Bächler (1940) erinnern (S. Brodar 1938). Die Holzkohlen stammen nach E. HofmANN von Zirbelkiefern- und Fichtenholz mit Jahresringen, die auf kurze Sommer und rauhes Klima hinweisen (Pinus cembra kommt heute in dieser Gegend nicht mehr vor). Viele Prähistoriker haben diese jungpaläolithische Station der großen Höhenlage wegen dem letzten Interglazial zugewiesen; aber mit Recht haben L. F. Zотz (1951), G. FreUnd (1952) und K. J. NARR (1954) sie in die große 1. Würmschwankung gestellt; denn wenn diese so viel länger gedauert hat als die ungefähr ebenso temperierte 
ca. 1000jährige Alleröd-Schwankung, muß das alpine Inlandeis ebenfalls bis über $1800 \mathrm{~m}$ geschwunden sein. Es ist daher sehr wahrscheinlich, daß auch das "alpine Paläolithikum" von E. BÄCHLER wenigstens zum größeren Teil in diese 1. Würmschwankung zu stellen ist (vgl. auch K. J. NARR 1954). Wahrscheinlich werden sich diese alpinen Bärenhöhlen, die of $t$ reich an Holzkohle sind, mit der $\mathrm{C}^{14}$-Methode datieren lassen.

In Norddeutschland sind mehrere Moorbildungen bekannt, die nach den Pollendiagrammen und der Stratigraphie würminterstadial sein dürften: 1. ein „Weichsel-Frühglazial“-Profil in Lüneburg (R. HALlik 1952), 2. an der schleswig-holsteinischen W-Küste $S$ vom Hindenburgdamm über Eem-Bildungen (E. DitTMER 1954), 3. bei Harksheide bei Hamburg (R. Hallik 1955), 4. im Hangenden des Loopstedter Eem-Interglazials (E. Kolumbe 1955), 5. Torfe im Hangenden von RW-Interglazialbildungen bei Schwindbeck, Orrel und Nedden-Averbergen in Niedersachsen (W. Selle 1952), 6. ? in Vechelde bei Braunschweig (W. Selle 1954). In allen diesen Fällen werden die Pollendiagramme von den Kurven der Kiefer und Birke beherrscht, in geringen Mengen kommen Fichte, Weide und Erle vor, sporadisch in Spuren auch die Hasel; der NBP erreicht öfters hohe Werte mit einem Minimum in der Mitte, weniger hohe der Ericales-Pollen; diese Befunde sprechen für eine \pm subarktische Pflanzendecke in Norddeutschland im 1 . Würminterstadial. In dieses gehört höchst wahrscheinlich auch das bekannte Interstadialvorkommen von Smidstrup in Jütland mit ebenfalls subarktischem Klima (K. Jessen 1928) mit Betula nana, Salix herbacea und Dryas octopetala oben, Pinus silvestris (Pollen), Juniperus communis, Betula pubescens, Empetrum, Geum, Rubus saxatilis, Potentilla palustris, ? Biber, Elch (Exkremente) in der Mitte und Dryas am Grunde, ferner das Interstadial von Schlutup bei Lübeck (P. Friedrich 1905), die nach meinem pollenanalytischen Befund unter subarktischen Klimaverhältnissen gebildete Diluvialkohle von Purmallen bei Memel, der Rixdorfer Horizont in Berlin (P. Woldstedt 1950), der ähnliche Horizont vom Fort Neudamm bei Königsberg, vielleicht auch die Interstadiale von Preetz bei Plön (E. W. Guenther 1951) und von Endingen W von Stralsund, die auch aus stratigraphischen Gründen doch wohl besser in diese Zeit als in ein Interstadial zwischen dem Pommerschen Stadium und dem Langeland-Vorstoß zu stellen sind, da der an beiden Fundorten festgestellte Elch das Vorhandensein von Wäldern in nicht zu großer Entfernung, also eine längere Wärmezeit voraussetzt. Es ist dieses aber nur eine Auswahl; nur mit Hilfe der $\mathrm{C}^{14}$-Methode kann mit Sicherheit ermittelt werden, ob diese fossilführenden Bildungen aus dem 1. Würminterstadial stammen.

Pedologisch unterscheidet sich, wie schon hervorgehoben, die Göttweiger Bodenbildung deutlich von der Paudorfer und Kremser (F. Brandtner 1954, J. Fink 1954, 1955). Die viel geringere Rotfärbung der Göttweiger Verlehmungszone und die viel geringere Verwitterung lassen auf ein merklich weniger warmes Klima schließen als zur Zeit der Entstehung der Kremser Verlehmungszone im RW-Interglazial. Leider scheint die Paläopedologie noch in den Kinderschuhen zu stecken. Die intakte Lößstruktur der Göttweiger Verlehmungszone im Gegensatz zur Kremser deutet nach F. BRANDTNER (1954) und J. FINK (1955) auf einen Steppenboden in einem temperierten kontinentalen mäßig humiden Klima mit Temperaturmitteln unter denen der postglazialen Wärmezeit; dichtere Baumbestände waren nach F. BRANDTNER damals nur an günstigen Standorten denkbar, lichte Wälder lediglich nur entlang der Flüsse und in feuchten Talniederungen. Nach Fr. ProŠEK \& V. LožEK (1955) ist die II. (die Göttweiger) fossile Verlehmungszone von Zamarovce eine Braunerde, also unter Wald entstanden; R. LaIs (1951) und Herr Prof. Dr. Dr. Mückenhausen (briefl. Mitteil. 1955) halten die Göttweiger Verlehmungszone in Nieder-Österreich auch für Braunerde. Die Herren Fr. ProšEK \& V. LožEK waren so liebenswürdig, mir folgende Auskunft zu erteilen: „Die fossilen Böden sind bei uns faziell entwickelt: in den heutigen Steppengebieten (Innerböhmen, Südmähren, Donau-Tiefland), wo heute die Schwarzerde eine führende Rolle spielt, sind auch die begrabenen Böden W 1/2 und RW als Schwarzerden entwickelt, die rißwürmische ist aber viel mehr 
degradiert. Dagegen im Waag-Gebiet, das eine ziemlich enge Tieflandbucht zwischen den Weißen Karpathen und dem Inovec-Gebirge bildet, ist das Klima feuchter, obwohl recht warm. So entstanden dort in den pleistozänen Stadialzeiten typische kalkige Lösse, aber in den warmen und hier recht feuchten Warmzeiten Braunerden, die in W 1/2 schwächer, in RW sehr stark entwickelt sind. Doch nach $S$ übergehen diese braunen Böden in normale Schwarzerden des Donau-Tieflandes. Der W 1/2-Boden in Moravany enthält eine typische Schneckenfauna der, Waldsteppe, die von der holozänen und rezenten Fauna kaum zu unterscheiden ist."

Hiernach und nach den paläofaunistischen und paläofloristischen Befunden ist $f$ ü $r$ die Göt tweiger Wärmeschwankung ein temperiertes, nicht vollin terg laziales Wa $1 \mathrm{dsteppenklim} \mathrm{a} \mathrm{anzunehmen,} \mathrm{das} \mathrm{im} \mathrm{humideren} \mathrm{nörd-}$ lichen Mitteleuropa merklich kühler als im südöstlichen, auch heute noch semi-ariden Teil war und in Westeuropa anscheinend nur eine schwache Spur im Lößprofil hinterlassen hat, während das Klima des RW-Interglazials eine sehr starke Bodenbildung („argile rouge“) hervorgebracht hat (vgl. F. E. Zeuner 1952, Fig. 60). D i e G ö t t w e i g e r W ä r m e schwankung war also nichtein Interglazial, sondern ein temperiertes Interstadial in der Würm-Eiszeit, wie es F. E. Zeuner (1952 S. 410) richtig charakterisiert hat; dieses Interstadial ist mit der Laufenschwankung von A. Penck und der Spiezer Schwankung von P. Beck synchron. Für die Benennung erscheint der Name Göt t w e ig e r Inter st a dial deswegen am zweckmäßigsten, weil die Göttweiger Leimenzone für die richtige Deutung dieser Wärmeschwankung ausschlaggebend war. F. E. Zeuner (1952) nennt sie L. Gl. 1/2, R. Grahmann (1952, 1956) "1. Würmschwankung“; diese Bezeichnungen sind ebenso wie der Name „Großes WürmInterstadial“ von L. WEINBERGER (1953) deswegen nicht zu empfehlen, weil die 2. oder kleine Würmschwankung L. Gl. 2/3 (das Paudorfer Interstadial) so sehr viel schwächer ist, daß eine $Z$ weiteilung der Würmeiszeit, wie sie neuerdings C. RathuENs (1951) vertritt, einer Dreiteilung entschieden vorzuziehen ist.

Die Länge des Göttweiger Interstadials muß sehr viel größer gewesen sein als die der ca. 1000jährigen Alleröd-Schwankung; letztere hat eine sehr geringmächtige Bodenbildung (über die leider nicht ausreichendes Zahlenmaterial vorliegt) hinterlassen, die erstere dagegen eine 50-100 cm mächtige Verlehmungszone. Vergleicht man diese mit der Mächtigkeit der rezenten Bodenbildung auf Löß im gleichen Gebiet, die ca. 12000-14000 Jahre alt sein dürfte, so erscheint in Anbetracht dessen, daß in die Bildungszeit der Göttweiger Verlehmungszone keine Wärmezeit vom Ausmaß der postglazialen fiel, eine Länge von ca. 15000 Jahren für das Göttweiger Interstadial, das sicher kürzer als das RW-Interglazial war, annehmbar. F. E. ZEUner (1952, S. 242) möchte ihm eine Länge von 30000 Jahren zuschreiben, aber auf Grund der Sonnenstrahlungskurve von Milankovitch. Bei einer solchen Länge eines temperierten Interstadials ist es erklärlich, daß seine Bodenbildung durch ihre Mächtigkeit ein Interglazial vortäuscht; von unvollständigen Bildungen des RW-Interglazials bei Abwesenheit von Leitfossilien wird es nur mit der $\mathrm{C}^{14}$-Methode zu unterscheiden sein. Bei einer Länge von ca. 15000 Jahren ist es durchaus anzunehmen, daß im Göttweiger Interstadial das alpine Inlandeis mindestens auf den gleichen geringen Umfang zusammengeschrumpft war wie kurz vor dem Alleröd-Interstadial; dafür spricht das Vorkommen von Eichen in Ablagerungen der Spiezer Schwankung und die mittelaurignacienzeitliche Kulturschicht in der Potočka-Höhle in $1700 \mathrm{~m} \mathrm{MH}$, und es ist durchaus möglich, daß auch die noch höher liegenden Stationen des „alpinen Paläolithikums“ in Bärenhöhlen dem Göttweiger Interstadial zuzuweisen sind.

F. E. Zeuner (1952, S. 202) stuft dieses Interstadial auf Grund der Sonnenstrahlungskurve in die Zeit zwischen 115000 und 72000 Jahren vor 1800 n. Chr. ein. Diese Einstufung ist nicht mehr haltbar, nachdem inzwischen für das Périgordien IV im Abri Pataud in Les Eyzies, Dordogne, $2 \mathrm{C}^{14}$-Bestimmungen ein Alter von $23600 \pm 800$ bzw. $24000 \pm$ 
1000 Jahren ergeben haben (Science 12 1, 1955, S. 487); für mitteleuropäische AurignacienFundplätze wird ein Alter von 23000-25000 Jahren angegeben (mündl. Mitteil. der Herren Prof. Dr. Schw abedissen und Dr. BrandTNER). Für eine Eem-Torfschicht sind in Groningen C14-Daten von ca. 50000 bis ca. 40000 ermittelt worden (briefl. Mitteil. von Herrn Prof. Dr. HL. DE VRIEs). Es muß also möglich sein, das Göttweiger Interstadial mit dieser Methode zu datieren und unter günstigen Umständen auch seine Länge zu ermitteln. Daher ist es dringend zu empfehlen, Holzkohlen für die $\mathrm{C}^{14}$-Datierung aus der Potočka-Höhle, aus Höhlen mit „alpinem Paläolithikum“ und aus Kulturschichten des ältesten mitteleuropäischen Aurignacien zur Verfügung zu stellen. In diesem Zusammenhang ist das $\mathrm{C}^{14}$-Datum „älter als 36000 Jahre“ für eine Torflinse 6 Fuß unter der Unterkante des Jüngeren Löß3 II (Hauptwürm) von Godarville in Belgien (zwischen Mons und Namur, wo leider Löß I fehlt) von Interesse; diese Torflinse war durch frostgestörte Sedimente vom Löß II im Hangenden und von einem Moustérien-Rastplatz mit Mammut und Nashorn im Liegenden getrennt (Science 12 1, 1955, S. 657). Nach diesem C14-Datum muß das RW-Interglazial doch lange vor 40000 vor heute geendet haben.

Von H. Gams (1935, 1954 und auf dem 4. INQUA-Kongreß in Pisa 1953) ist die Ansicht vertreten worden, daß der Moustier-Vorstoß der Würmeiszeit, also Altwürm, die kalte Phase (nach H. Gams 1954 Präwürm) der Herning-Profile und das Göttweiger Interstadial die temperierte Phase $\mathrm{d}$.h. die 2. Wärmezeit (= F-Interglazial) der RWinterglazialen Herning-Profile gewesen sei, deren 1. Wärmezeit mit dem Wärmemaximum die Eem-Phase gewesen ist. Dagegen spricht aber die Tatsache, daß das Altwürm mit dem Moustérien in W-Frankreich jünger als die marine Transgression Monastir II ist (die der 2. Wärmezeit der Herning-Profile entsprechen dürfte), daß hohe Werte von Carpinus und Corylus, wie sie bisweilen in der 2. Wärmezeit der Herning-Profile festgestellt worden sind, für das Göttweiger Interstadial noch nicht nachgewiesen sind; mit P. WOLDSTEDT (1950) ist auch mit der Möglichkeit zu rechnen, daß in den Herning-Profilen die Zweiteilung der Wärmezeit durch eine periglaziale Schichtenstörung zu Beginn der Würmeiszeit vorgetäuscht ist.

Es erhebt sich nun die Frage, wie weit der Moustier-Vorstoß (Altwürm) gereicht hat. In Norddeutschland vermutet P. WOLDSTEDT als äußerste Südgrenze dieser Vereisung einen von der Hauptwürmvereisung überfahrenen Endmoränenzug im Gebiet von Stettin (Rosenthaler Randlage); möglicherweise gilt das auch für die westliche Umrandung der Lübecker Bucht (P. Woinstedt 1950, S. 44, 213, 310). E. DitTMER (1954, S. 175) schließt aus der Stratigraphie eines nordfriesischen Vorkommens von würmzeitlichen Interstadialtorfen, daß das nordische Inlandeis auch im ersten Würmvorstoß (Altwürm) auf schleswigholsteinischem Gebiet gelegen haben müsse. In England (East Anglia) könnte vielleicht die Little Eastern Glaciation der Altwürm-Phase entsprechen (vgl. F. E. Zeuner 1952, S. 189, 190), im Ristinge Klint auf Langeland die untere der beiden deutlich getrennten Geschiebemergelbänke (vgl. P.WoldstedT 1950, S. 59). Daß dieses 1. Würm-Stadial mit dem Warthe-Stadium identifiziert werden kann, wie es mit W. SoErgel z. B. H. Freising (1949, S. 22) und F. E. Zeuner (1952, z. B. S. 118, 132, 155) getan haben, ist nach P. WoldSTEDT (1954) unmöglich, da das Warthe-Stadium die letzte Phase der Saale-(Riß-)Eiszeit ist.

Im nördlichen Voralpengebiet hat ebenfalls der erste Würm-Vorstoß nicht ganz die maximale Ausdehnung des letzten erreicht. Diese Auffassung, die sich auf den Nachweis überfahrener Endmoränen stützt, ist bekanntlich zuerst von B. EBERL und J. KNAUER vertreten, jedoch von anderen abgelehnt worden; aber nach neueren Untersuchungen gibt es im Alpenvorland tatsächlich ältere Würm-Moränen, die von jüngeren Vorlandgletschern überfahren worden sind, welche die äußersten Jungmoränen gebildet haben (E. EBERS 1955, S. 102-104, E. Ebers \& L. WeINBERger 1954, L. WeINBERger 1953, 1955); weitere Beweise für eine Altwürmphase haben C. Rathjens (1951) und E. Kraus (1955) mitgeteilt. Besonders überzeugend ist das Profil vom Glütschtal mit Schieferkohle (P. BECK 1938, 
1954, 1955 und C. Rathjens 1951, Fig. 6); hier hatte aber die Altwürm-Vereisung eine größere Ausdehnung als die Hauptwürm-Vereisung. Sehr beachtenswert ist ferner das teilweise schon von A. PENCK \& E. BRÜCKNER beschriebene Vorkommen von verknetetem Löß an der Basis von Hauptwürm auf dem östlichen Salzachgletscherflügel in Oberösterreich bei Gundertshausen unmittelbar im Hangenden der Rißmoränen und Hochterrassenschotter; A. PENCK \& E. BRÜCKNER erwähnen ein Gegenstück hierzu für das Gebiet von Lyon im Rhône-Gletschergebiet (E. EBERs 1955, S. 102).

Die zweite Frage in diesem Zusammenhang ist die nach der Größe des Inlandeisrückzugs im Göttweiger Interstadial. Wenn im südlichen und noch mehr im südöstlichen Mitteleuropa in dieser Zeit (im Optimum dieser Wärmeschwankung) schon Vorposten wärmeliebender Holzarten in Tieflagen erschienen sind, so ist zu vermuten, daß sich der S-Rand des skandinavischen Inlandeises mindestens ebenso weit wie im Alleröd-Interstadial nach N, d.h. bis Mittelschweden und Südfinnland, zurückgezogen hatte. Fichtenholz aus der Moräne bei Malung (ca. $61^{\circ} \mathrm{N}$ ) und Boliden (ca. $65^{\circ} \mathrm{N}$ ) in Schweden ist nach einer $\mathrm{C}^{14}$ Bestimmung in Stockholm ca. 23000 Jahre alt (nach freundl. briefl. Mitteil. von Frau Prof. E. H. De GeEr 1955); da dies Datum sicher zu jung ist, müßte es in einem $C^{14}$ Laboratorium mit größerer Erfahrung nachgeprüft werden. Dafür, daß das Inlandeis in den Alpen bis über $1800 \mathrm{~m} \mathrm{M} \mathrm{H}$ wie im Alleröd-Interstadial geschwunden sein muß, sprechen die Befunde in der Potočka-Höhle und im Glütschtal bei Thun in der Schweiz.

Vergleicht man die pollenanalytischen Befunde nordwestdeutscher würminterstadialer Moorbildungen mit denen der im gleichen Gebiet untersuchten Eem-Schichten, so ist es klar, daß die ersteren wirklich nicht interglazial genannt werden können und daß zur Zeit ihrer Bildung noch ein erheblicher Rest des skandinavischen Inlandeises (wohl so groß wie bei der Bipartition) vorhanden gewesen sein muß. Di e Gö t t w e ig e r Wär m e $s c h w a n k u n g s c h e i d e t$ also $2 \mathrm{Stadien} \mathrm{der} \mathrm{Würm-Vereisung,} \mathrm{ist}$ alsoein Interstadialder Würm-Eiszeit.

Zur Benennung dieser beiden Würm-Stadien sollten die Bezeichnungen W I und W II endlich nicht mehr verwendet werden, da sie von den einzelnen Geologen und Prähistorikern seit langem in ganz verschiedenem Sinne gebraucht worden sind. Am besten sind die Namen A l t w ü rm (R. Grahmann, L. Weinberger und E. Kraus) oder F r üh w ü r m (E. Ebers, P. Woldstedt). Der Name Jungriß ist entschieden abzulehnen. F. WeiDENBACH (1955), der wie G. Götzinger und 1955 in Wien H. Freising und J. Fink die Göttweiger Bodenbildung fälschlich dem RW-Interglazial zuweist, stellt die nächst ältere, die Kremser Bodenbildung, in ein Interglazial Mittelriß-Jungriß, was ganz unmöglich ist, da das Interglazial mit der letzten Antiquus-Fauna in Holland und Norddeutschland einwandfrei jünger als die Riß-Eiszeit, also als RW-Interglazial zu bezeichnen ist (P. WoLDSTEDT 1954). Viel weniger bedenklich, als die von A. PENCK als einheitlich angenommene Riß-Eiszeit durch ein Vollinterglazial (die Kremser Bodenbildung des RW-Interglazials im Sinne von A. Penck) zu unterbrechen, ist es, die von A. PencK im Riß-Tal als „klassisches Riß “ ausgeschiedenen Terrassen mit nur e i n e m Löß und schwächerer Verwitterung als beim Hochterrassenschotter dem Würmkomplex als obere Niederterrasse zuzuweisen; diese Ansicht vertritt auch Herr Prof. Dr. R. Grahmann (briefl. Mitteil.). A. Penck hat sein mit E. BRücKNER verfaßtes grundlegendes Werk über die Alpen im Eiszeitalter nicht als quartärgeologische Bibel betrachtet, denn er hat manche der darin veröffentlichten Ansichten auf Grund neuen Beobachtungsmaterials später revidiert. Die heute im Alpenvorland arbeitenden Geologen sollten unvoreingenommen prüfen, ob in der Würm-Eiszeit wirklich nur e i n e Niederterrasse gebildet worden ist. Eine höhere NT ist offenbar z. B. die von L. WeInberger $(1955$, S. 18, 27) im Gebiet des Salzachgletschers zwischen der NT und HT festgestellte Zwischenterrasse, die nach ihm mit großer Wahrscheinlichkeit dem inneren überfahrenen Würm-Moränenwall entspricht (vgl. auch E. EBERs \& L. WEINBERGER 1954 und Verhandl. d. Geol. Bundes-Anst. Wien, Sonderheft D, 1955, S. 41 und 
Gliederung der letzten Eiszeit

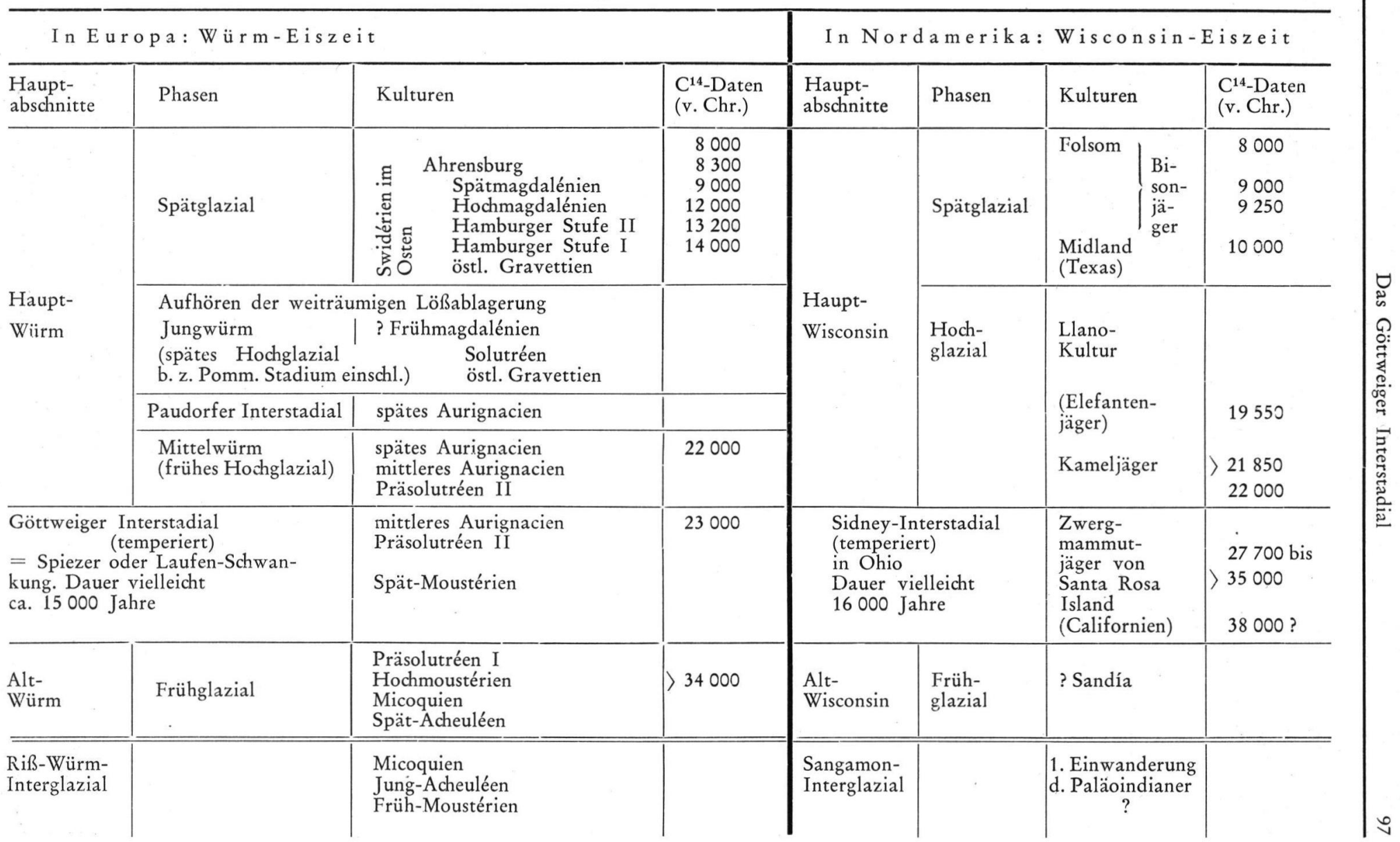


54). Es ist mit der Möglichkeit zu rechnen, daß vielfach im 2. Würm-Stadial, das mit einer erheblich stärkeren und länger dauernden Klimadepression verbunden war, durch stärkere Aufschotterung die Spuren einer interstadialen Erosion der Aufschotterung der ersten Phase beseitigt worden sind.

Dafür, daß die Altwürm-Vereisung kürzer war und mit einer merklich geringeren Klimadepression als das zweite Würm-Stadial verbunden war, sprechen verschiedene Befunde: ihre Ausdehnung ist (mit Ausnahme des Schweizer Mittellandes und des südlichen Alpenvorlandes in Osterreich) kleiner gewesen, der Altwürm-Löß hat eine viel geringere Mächtigkeit, seine Striata-Fauna (Leitform: Helicella striata Müll.) ist eine typische "warme" Schneckenfauna der Lößsteppe, die im Waag-Gebiet für diesen Löß bezeichnend ist (Fr. ProšEK \& V. LožEK 1955), die lehmreiche Frostbruchschicht dieser Zeit in den Höhlen hat in der Regel eine viel geringere Mächtigkeit, und ihre Kalkbrocken sind kantengerundet (L. F. Zotz 1955).

Die zweite Phase der Würm-Vereisung ist die „einheitliche Würmeiszeit“ im Sinne von A. Penck (seit 1920), C. Troll, J. Büdel und F. Weidenbach. Höhlenablagerungen dieser Art lassen öfters in einer dünnen, stärker verwitterten Schicht eine schwache Klimabesserung erkennen, die mit dem schwachen Paudorfer Interstadial parallelisiert wird, welches im Löß semi-arider Gebiete im südöstlichen Mitteleuropa als schwache Bodenbildung registriert ist; die Begleitfauna des späten Aurignacien von Předmost, das in diese Zeit fällt, ist auffallend reich an Waldtieren des gemäßigten Klimas (L. F. Zotz 1951). Diese 2. Wärmeschwankung war aber im Vergleich zur Göttweiger so schwach, daß es nicht zweckmäßig erscheint, die zweite Phase in Mittel- und Spätwürm aufzuteilen, sondern sie als $\mathrm{H}$ a u p t w ü r m mit den Abschnitten Mittelwürm, Spätwürm und Spätglazial zu bezeichnen. Daß dieses zweite Würm-Stadial erheblich länger und wenigstens zeitweilig (im Aurignacien nach Ansicht namhafter Paläozoologen) kälter als das erste war, geht aus der Stratigraphie des Lösses (mit der typischen „kalten“ Columella-Fauna: Leitform: Columella edentula columella Mart. nach Fr. ProšEK \& V. LožEK 1955) und der Höhlenschichten hervor. Für besonders starke Klimadepressionen im Hauptwürm-Stadial gelten die neueren Vegetationskarten der Würmeiszeit, z. B. von J. Büdel (1950), B. FrenzeL \& C. Troll (1952) und P. Woldstedt (1954 b). Das Maximum von Hauptwürm war das Brandenburger Stadium, im Alpenvorland die äußerste Jungendmoräne.

Die Klimakurve der Würm-(Weichsel-)Eiszeit, wie sie P. Woldstedt (1954, S. 43 und in diesem Jahrbuch S. 82) angegeben hat, bedarf jetzt m. E. einer Abänderung: die Kurve des Göttweiger Interstadials dürfte ungefähr die gleiche Höhe wie die des Alleröd-Interstadials erreichen müssen, aber (mit Schwankungen) ein Intervall umspannen, das ca. 15000 Jahre mißt.

Die hier vorgetragene Auffassung wird durch die wichtige, auf ca. $50 \mathrm{C}^{14}$ - Daten basierende Arbeit von R. F. Flint \& M. Rubin (1955) über die Stadien der letzten Vereisung (Wisconsin) Nordamerikas in der östlichen Hälfte der USA gestützt. Eine Parallelisierung mit der Gliederung der Würm-Eiszeit in Europa (vgl. hierzu E. H. De GeER 1955) ist zweifellos zulässig, da die säkularen Klimaschwankungen des Pleistozäns auf kosmische Ursachen zurückzuführen sind und bereits nachgewiesen ist, daß dem AllerödInterstadial und dem Salpausselkä-Stadium Europas das Two Creeks Forest Bed-Interstadial und das Mankato-Stadium (auf Grund von mehreren C14-Datierungen) in Nordamerika entsprechen. Die Wisconsin-Vereisung, die nach Ablagerung des Farmdale-Lößes (vor ca. 28000 Jahren) das Gebiet der Großen Seen erfaßte (das N-Ufer des Erie-Sees wurde vor $27500 \pm 1200$ Jahren erreicht) und ihre maximale Ausdehnung zwischen 20000 und 18000 Jahren vor heute erreichte, entspricht der europäischen Hauptwürm-Vereisung; für ihr Maximum, das Brandenburger Stadium, hält P. WoldstedT (1954 b, S. 341) die Datierung 20000 Jahre vor heute für durchaus annehmbar. Das Hauptwisconsin-Eis überfuhr vor $23000 \pm 800$ Jahren bei Sidney in Ohio eine ältere mit Fichten bewaldete 
Grundmoräne, deren auch anderwärts in Ohio festgestellter Boden nicht einem Gumbotil ähnelt, also nicht interglazial sein kann und auch von R. F. Flint \& M. Rubin (1955) für jünger als letztinterglazial (Sangamon) gehalten wird. Daher muß diese ältere Grundmoräne nach dem Sangamon von einem Altwisconsin-Eis (entsprechend dem Altwürm Europas) abgelagert worden sein. Alt- und Hauptwisconsin sind durch ein Interstadial mit Mooren und Nadelwäldern getrennt, dessen Länge auf Grund der Mächtigkeit der Verwitterungsrinde, also des begrabenen Bodens (1,22 $\mathrm{m}$ verglichen mit einer Verwitterungsrinde von $0,76 \mathrm{~m}$ auf der 10000 Jahre alten Mankato-Grundmoräne; die Verwitterungsrinde auf der nach einer $\mathrm{C}^{14}$-Datierung ca. 14000 Jahre alten HauptwisconsinGrundmoräne bei Sidney, Ohio, ist $1,10 \mathrm{~m}$ dick) auf rund 16000 Jahre geschätzt worden ist! In diesem Sidney-Interstadial sind wohl (spätestens) die ersten Einwanderer aus Nordostasien nach Nordamerika gelangt. Eine Gyttja aus einer Nadelwaldzeit am N-Ufer des Erie-Sees ist älter als 33000 Jahre.

Es kann also jetzt kein Zweifel mehr an der Richtigkeit der in neuerer Zeit besonders von C. Rathjens (1951) vertretenen Auffassung bestehen, daß in der letzten Eiszeit in Europa (und Nordamerika) zwei weitgehend selbständige Stadien durch ein langes temperiertes (im $\mathrm{N}$ kühleres) Interstadial (in Europa: Göttweiger Interstadial, in Nordamerika: Sidney-Interstadial) getrennt waren. Dieses Interstadial ist also ein Leithorizont der letzten Eiszeit in Europa und Nordamerika wie das Alleröd-Interstadial (H. Gross 1954).

\section{Schrift tu m}

Abkürzungen: DGU = Danmarks Geolog. Undersøgelse, E. u. G. = Eiszeitalter und Gegenwart, INQUA $=$ Verhandl. (Actes) der Internat. Quartärvereinigung.

Bächler, E. (1940) Das Alpine Paläolithikum der Schweiz. 2 Bde. Basel.

Bayer, J. (1927) Der Mensch im Eiszeitalter. Wien. - - (1929) Die Olschewakultur, eine neue Fazies des Schmalklingenkulturkreises in Europa. - Eiszeit und Urgeschichte 6.

Beck, Paul (1938) Bericht über die außerordentliche Frühjahrsversammlung der Schweizerischen Geolog. Gesellschaft in Thun 1938. - Eclogae geolog. Helvetiae 31, 173-198. - - (1954) Regionale Grundlagen für die Gliederung des alpinen Quartärs. - Ibidem 47, 369-373. - - (1955) Quartärablagerungen im Querschnitt durch die zentralen Schweizeralpen. INQUA IV Rom-Pisa 1953.

BrandtNer, F. (1949) Die bisherigen Ergebnisse der stratigraphisch-pollenanalytischen Untersuchung eines jungeiszeitlichen Moores von interstadialem Charakter aus der Umgebung von Melk a. d. Donau, Nieder-Ósterreich. - Archaeologia Austriaca 2, 5-32. - - (1950) Uber die relative Chronologie des jüngeren Pleistozäns Nieder-Ơsterreichs. - Ibidem 5, 101-113. - - (1954) Jungpleistozäner Löß und fossile Böden in Nieder-Ơsterreich. E. u. G. $4 / 5,49-82$.

Bohmers, A. (1939) Die Ausgrabungen in den Höhlen von Mauern. - Forschungen u. Fortschritte 15. - - (1944) Die Mauerner Höhlen und ihre Bedeutung für die Einteilung der Altsteinzeit. - „Das Ahnenerbe“, Bericht über die Kieler Tagung 1939. Neumünster.

Brodar, S. (1938) Die Potočka-Höhle $(1700 \mathrm{~m})$, die höchste jungpaläolithische Station in den Alpen. - INQUA III Wien 1936, 314-315.

Brunnacker, K. (1956) Die Höhlensedimente im Hohlen Stein bei Schambach. - Geol. Bl. NOBayern 5, 21-32.

Bübel, J. (1950) Die Klimaphasen der Würmeiszeit. - Die Naturwiss. 3\%, 438-449. - - (1953) Die „periglazial“-morphologischen Wirkungen des Eiszeitklimas auf der ganzen Erde. Erdkunde \%, 249-266.

De Geer, E. H. (1955) La déglaciation américaine selon la chronologie De Geer. - Bull. Soc. géol. France (6) 5, 423-432.

Dittmer, E. (1954) Interstadiale Torfe in würmeiszeitlichen Schmelzwassersanden Nordfrieslands. - E. u. G. 4/5, 172-175.

Ebers, E. (1955) Hauptwürm, Spätwürm, Frühwürm und die Frage der älteren Würmschotter. E. u. G. 6, 96-109.

Ebers, E. \& Weinberger, L. (1954) Die Periglazial-Erscheinungen im Bereich und Vorfeld des eiszeitlichen Salzach-Vorlandgletschers im nördlichen Alpenvorland. - Göttinger Geogr. Abh. 15. 
FINk, J. (1954) Die fossilen Böden im österreichischen Löß. - Quartär 6, 85-107. - - (1955a) Prinzipielle Fragen bei der Erforschung fossiler Böden im (österreichischen) Löß. INQUA IV Rom-Pisa 1953. - - (1955b) Verlauf und Ergebnisse der Quartärexkursionen in Österreich 1955. - Mitteil. geogr. Ges. Wien 97, 209-216.

Flint, R. F. \& Rubin, M. (1955) Radiocarbon Dates of Pre-Mankato Events in Eastern and Central North America. - Science 121, 649-658.

Freising, H. (1949) Lösse, Fließerden und Wanderschutt im nördlichen Württemberg. - Diss. T. H. Stuttgart. - - (1951) Neue Ergebnisse der Lößforschung im nördlichen Württemberg. Jahresh. geol. Abt. württ. Statist. L.-A. 1, 54-59.

Frenzel, B. \& Troll, C. (1952) Die Vegetation des nördlichen Eurasiens während der letzten Eiszeit. - E. u. G. 2, 154-167.

Freund, Gisela (1952) Die Blattspitzen des Paläolithikums in Europa. - Quartär-Bibliothek Bd. 1, Bonn.

Friedrich, P. (1905) Die Grundmoräne und die jungglacialen Süßwasserablagerungen der Umgebung von Lübeck. - Mitt. geogr. Ges. u. naturhistor. Museums Lübeck, 2. Reihe, H. 20 .

Gams, H. (1935) Beiträge zur Mikrostratigraphie und Paläontologie des Pliozäns und Pleistozäns von Mittel- und Osteuropa und Westsibirien. - Eclogae geol. Helvet. 28, 1-31. - Neue Beiträge zur Vegetations- und Klimageschichte der nord- und mitteleuropäischen Interglaziale. - Experientia 10, 357-363.

Götzinger, G. (1936) Das Lößgebiet um Göttweig und Krems an der Donau. - INQUA III Wien 1936, Führer für die Quartär-Exkursionen in Ósterreich, I. Teil, 1-11. - - (1938) Exkursion am 7. und 8. September 1936 in das Lößgebiet des niederösterreichischen Weinviertels und angrenzenden Waldviertels. - INQUA III Wien, 335-338.

Götzinger, G. \& Hassinger, H. (1938) Exkursion am 3. September 1936, Wien-HerzogenburgGöttweig-Spitz-Krems-Wien. - INQUA III Wien 325-328.

GrahmanN, R. (1937) In: Die dritte Internationale Quartär-Konferenz (INQUA) und ihre Belehrungsreisen in Ơsterreich, September 1936. - Z. f. Gletscherk. 25, 241-280. - - (1952, 1956) Urgeschichte der Menschheit. Stuttgart, 1. und 2. Auflage.

Gross, H. (1954) Das Alleröd-Interstadial als Leithorizont der letzten Vereisung in Europa und Amerika. - E. u. G. 4/5, 189-209.

Guenther, E. W. (1951) Ein eiszeitlicher Elch aus Preetz und die Frage eines Weichselinterstadials in Ost-Holstein. - Schr. natw. Ver. Schleswig-Holstein 25, 115-124.

HaLliK, R. (1952) Ein „Weichsel-Frühglazial“-Profil in Lüneburg. - E. u. G. 2, 168-172. - - (1955) Uber eine Verlandungsfolge weichsel-interstadialen Alters in Harksheide bei Hamburg. E. u. G. 6, 116-124.

Iversen, Johs. (1954) The Late-Glacial Flora of Denmark and its Relation to Climate and Soil. DGU II. R. Nr. 80, 87-119.

Jessen, K. (1928) in V. Madsen u. Mitarb.: Übersicht über die Geologie von Dänemark. - DGU, V. R., Nr. 4.

Jessen, K. \& Milthers, V. (1928) Stratigraphical and Paleontological Studies of Interglacial Fresh-Water Deposits in Jutland and Northwestern Germany. - DGU, II. R., Nr. 48.

Kolumbe, E. (1955) Uber interglaziale und interstadiale Bildungen von Loopstedt am Haddebyer Noor bei Schleswig. - E. u. G. 6, 39-40.

Kühnelt, W. (1938) Die quartären Mollusken OOsterreichs und ihre paläoklimatische Bedeutung. INQUA III Wien 1936, 234-236.

Kürper, H. (1955) Ausblick auf das Pleistozän des Raumes von Wien. - Verhandl. geol. Bundesanstalt Wien, Sonderheft D: Beitrag z. Pleistozänforschung in Österreich, 136-152, Tafel XI.

LAIS, R. (1941). UUber Höhlensedimente. - Quartär 3, 56-108 - - (1951) Über den jüngeren Löß in Niederösterreich, Mähren und Böhmen. - Ber.naturf.Ges. Freiburg i. Br. 41, 119-178.

Lehmann, U. (1954) Die Fauna des „Vogelherds“ bei Stetten ob Lontal (Württemberg). - N. Jb. Geol. u. Pal., Abh. 99, 33-146.

LüDI, W. (1953) Die Pflanzenwelt des Eiszeitalters im nördlichen Vorland der Schweizer Alpen. Veröff. geobot. Inst. Rübel in Zürich, H. 27, Bern.

Mottl, Maria (1938) Faunen und Klima des ungarischen Moustérien. - INQUA III Wien 1936, 248-251.

NARR, K. J. (1953) Riß oder Würm? - Germania 31, 125-134. - - (1954) Formengruppen und Kulturkreise im europäischen Paläolithikum. - 34. Ber. Röm.-German. Komm. f. 1951$1953,1-40$.

Nehring, A. (1890) Úber Tundren und Steppen der Jetzt- und Vorzeit. Berlin.

ProšEk, Fr. \& LožeK, V. (1955) Výzkum sprasového profilu v Zamarovcích u Trenčina (Untersuchung des Lößprofils von Zamarovce bei Trenčin). - Anthropozoikum 4, 1954, 181212. Prag.

Rathjens, C. (1951) Uber die Zweiteilung der Würmeiszeit im nördlichen Alpenvorland. - Peterm. geogr. Mitteil. 95, 89-97. 
RIEK, G. (1934) Die Eiszeitjägerstation am Vogelherd im Lonetal. Bd. I. Die Kulturen. Tübingen.

Schmucker, Th. (1942) Die Baumarten der nördlich gemäßigten Zone und ihre Verbreitung. Silvae orbis.

SснütrumpF, R. (1944) Die pollenanalytische Datierung der altsteinzeitlichen Funde von Mauern. - Ber. über die Kieler Tagung d. Forschungs- und Lehrgemeinschaft „Das Ahnenerbe“ 1939, 74-79, Neumünster.

SELle, W. (1952) Die Interstadiale der Weichselvereisung. - E. u. G. 2, 112-119. - - (1954) Das Vechelder Interstadial. - E. u. G. 4/5, 176-108.

Soergel, W. (1919) Löße, Eiszeiten und paläolithische Kulturen. Jena. - - (1939) Das diluviale System. Berlin.

VahL, M. (1902) De qvartaere Stepper i Mellemeuropa. - Geograf. Tidsskr. Kopenhagen.

WeIDENBACH, F. (1952) Gedanken zur Lößfrage. - E. u. G. 2, 25-36. - - (1955) Zeitliche Einordnung der jungpleistozänen Ablagerungen Mitteleuropas. - INQUA IV Rom-Pisa 1953.

Weinberger, L. (1955) Exkursion durch das österreichische Salzachgletschergebiet und die Morä-

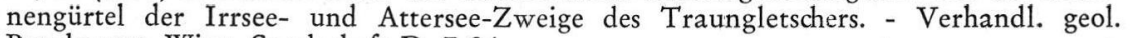
Bundesanst. Wien, Sonderheft D, 7-34.

WetzeL, R. (1954) Quartärforschung im Lonetal. - E. u. G. 4/5, 106-141.

WoldSTEDT, P. (1950) Norddeutschland und angrenzende Gebiete im Eiszeitalter. - Stuttgart. - (1952) Probleme der Terrassenbildung. - E. u. G. 2, 36-44 - - (1954a) Saaleeiszeit, Warthestadium und Weichseleiszeit in Norddeutschland. - E. u. G. 4/5, 34-48. - (1954b) Das Eiszeitalter. 2. Aufl. 1. Bd. Stuttgart.

Zeunfr, F. E. (1952) Dating the Past. An Introduction to Geochronology. 3rd ed. London. (1954) Riss or Würm? - E. u. G. 4/5, 98-105.

Zотz, L. F. (1951) Altsteinzeitkunde Mitteleuropas. Stuttgart. - - (1955) Das Paläolithikum in den Weinberghöhlen bei Mauern. - Quartär-Bibliothek Bd. 2. Bonn.

Manuskr. eingeg. 23. 4. 1956.

Anschrift d. Verf.: Stud. prof. Dr. Dr. h. c. Hugo Groß, Bamberg, Kunigundendamm 59. 